\title{
A PROBABILISTIC APPROACH TO THE PROBLEM OF ASSESSING THE EFFICIENCY OF THE TRANSFORMED VEGETATION INDEX
}

\author{
G.A. SKIANIS ${ }^{1}$, D. VAIOPOULOS ${ }^{1} \&$ K. NIKOLAKOPOULOS ${ }^{2}$ \\ ${ }^{1}$ Remote Sensing Laboratory, Department of Geology and Geoenvironment, University of Athens, Greece. \\ ${ }^{2}$ Institute of Geological and Mineral Exploration, Athens, Greece.
}

\begin{abstract}
Vegetation indices are usually defined and evaluated empirically, according to their performance on images of areas with certain vegetation types and targets of interest. In this paper we propose a probabilistic approach to the problem of assessing the efficiency of a vegetation index, more precisely the transformed vegetation index (TVI), in its two versions (TVIa and TVIb). A proper distribution is introduced in order to describe the histograms of the red and near infrared channels. Then, the mathematical expressions for the distribution of the TVIa and TVIb values are derived, according to theorems of statistics. The study of the behavior of this distribution shows that the standard deviation of TVIa is bigger than that of the more often employed normalized differences vegetation index (NDVI). This theoretical prediction is verified using satellite images of various regions in Greece and in the Mediterranean Sea. The signal to noise ratio of TVIa and TVIb images is also studied and it is shown that this ratio is bigger than that of NDVI, if the brightness value in the near infrared channel is considerably bigger than that of the red channel. The general conclusion is that TVIa produces images with a good contrast and TVIb presents a good signal to noise ratio over areas with a rich vegetation cover.
\end{abstract}

Keywords: NDVI, signal to noise ratio, standard deviation, TVI, TVIa, TVIb.

\section{INTRODUCTION}

In geological and environmental research, spectral band ratios are often used as vegetation indices, for mapping the vegetation cover of the area under study. Various vegetation indices have been introduced, mainly based on empirical criteria of response over vegetation types, soils or geological targets of interest [1-10]. Great effort has been used in extracting information about vegetation cover parameters such as the leaf area index (LAI) or the forest biomass, from vegetation indices and various reflectance bands [11-24]. Attempts to assess crop yield using vegetation indices have also been made [25, 26]. In these approaches, remote sensing data are compared with ground data and correlation coefficients are computed. In certain cases, the sensitivity of various vegetation indices is assessed with the aid of mathematical models which associate the vegetation cover with its reflectance at various bands [27-30].

Recently, we have developed an alternative methodology of assessing the efficiency of vegetation indices with the aid of probability theory, which has already been applied for the study of the normalized differences vegetation index (NDVI) and the simple vegetation index [31], as well as for the soil adjusted vegetation index [32]. The methodological difference between this approach and other efforts is that our research is centered on the statistical behavior of the vegetation index under study, using theorems about bivariate distributions and appropriate density functions to describe the histograms of the frequency bands. This means that the whole approach is focused on the mathematical structure of the function which describes the vegetation index and not on its functionality at different ground types or its correlation with biophysical parameters.

(C) 2007 WIT Press, www.witpress.com

ISSN: 1743-7601 (paper format), ISSN: 1743-761X (online), http://journals.witpress.com

DOI: 10.2495/SDP-V2-N4-461-480 
In this paper we study the behavior of the transformed vegetation index (TVI) [3], using this probabilistic approach. TVI is usually defined in two forms, TVIa and TVIb. TVIa is given by [3, 12]:

$$
u=\left\{\begin{array}{ccc}
\sqrt{\frac{x-y}{x+y}} & \text { for } & x \geq y, \\
0 & \text { for } \quad x<y,
\end{array}\right.
$$

where $x$ and $y$ are the brightness values at the near infrared (NIR) and red channel respectively and $u$ is the value of the vegetation index.

In order to reduce the number of pixels with a zero value, the modified version of TVI, i.e. TVIb, is often preferred, which is given by the relation [17,33]:

$$
u=\left\{\begin{array}{ccc}
\sqrt{\frac{x-y}{x+y}+0.5} & \text { for } & x \geq y / 3 \\
0 & \text { for } & x<y / 3
\end{array}\right.
$$

The most frequently used vegetation index is NDVI ([34-37] and many others). NDVI is defined by [2]:

$$
u=\frac{x-y}{x+y} .
$$

Although TVI has not been extensively used in remote sensing [3, 12, 17, 38, 39], we find that from the methodological point of view it is interesting to apply the probabilistic approach in order to study its behavior. After all, according to Rahman et al. [17], TVI presents a better correlation with biomass content than the frequently used NDVI; therefore, it is worthwhile to draw a little bit more attention to this index.

In this paper we compare the statistical parameters and the qualitative features of the TVIa, TVIb and NDVI images. The quantitative evaluation of these three vegetation indices is mainly based on the values of the standard deviation and the signal to noise ratio of the images. An image of a vegetation index with a large standard deviation has a good contrast, which may help in detecting targets with a different tonality. A narrow vegetation index histogram (with a small standard deviation) may be broadened by histogram stretching. However two pixels with the same initial tonality can not have a varied tonality in the histogram stretched image, even if they represent different land cover types. On the other hand, if further processing of the vegetation index images (e.g. monitoring temporal changes in land cover) is supposed to take place, histogram stretching should be avoided since it distorts the original values of the pixels.

A large signal to noise ratio means an image with a reduced noise, limited tonality differences between adjacent pixels in the same land cover unity and sharp edges between adjacent formations in such a way that the targets of interest may be expressed more clearly.

Introducing proper distributions and taking into account certain theorems of statistics, we study the characteristics of the histograms of the three vegetation indices (NDVI, TVIa and TVIb). We test the theoretical predictions with real data obtained by a Landsat 7 satellite image from an island off western Greece and a SPOT vegetation image from the Mediterranean Sea. The results and conclusions of this paper may be useful in mapping more clearly the differences in the vegetation cover.

\section{THE PROBABILISTIC APPROACH}

In order to study the statistical characteristics of TVIa and TVIb, the histograms of the $x$ and $y$ channels have to be simulated by a proper distribution. It is reasonable to assume that this distribution is zero for a null brightness value, presents a peak at a relatively low value of the tonality range and gets 
practically nullified at high brightness values [40]. A satellite image, from which the atmospheric scattering has been removed, is expected to have such a behavior, if histogram stretch has not been done. In practice, the behavior of the histogram may be more complicated and it may present more than one peak, but it is reasonable to make a rough approximation of the histogram by a simple distribution, which may help in the mathematical analysis.

Vaiopoulos et al. [31] have proposed the following distributions $p_{1}$ and $p_{2}$ in order to describe the histograms of the channels $x$ and $y$, respectively:

$$
\begin{aligned}
& p_{1}(x)=2 a_{x} x \cdot \mathrm{e}^{-a_{1} x^{2}}, \\
& p_{2}(y)=2 a_{y} y \cdot \mathrm{e}^{-a_{2} y^{2}},
\end{aligned}
$$

where $a_{x}$ and $a_{y}$ are positive parameters that control the prevailing value and the standard deviation of the histogram [31]. The range of the brightness values $x$ and $y$ is from 0 to 255. In Fig. 1 the behavior of $p_{1}$ and $p_{2}$ is graphically presented. It can be observed that the distributions $p_{1}$ and $p_{2}$ present the behavior which is assumed for a histogram (a peak at a relatively low tonality and practically null frequencies at high brightness values). They also present a positive skewness which appears quite often in digital images [40]. In many cases, the image histogram is approximated by a Gaussian distribution [41], but this distribution may work efficiently if the pixels have similar characteristics. In a more general case, the positive skewness should be taken into account.

If $x$ and $y$ brightness values are not correlated, the combined distribution $f(x, y)$ of the tonalities of $x$ and $y$ channels is the product $p_{1}(x) \times p_{2}(y)$, which means that:

$$
f(x, y)=4 a_{x} a_{y} \cdot \mathrm{e}^{-a_{x} x^{2}-a_{y} y^{2}} .
$$

The assumption of a zero correlation between $x$ and $y$ may help in avoiding technical complications and work, as much as possible, with easy to use mathematical expressions. On the other hand, this assumption is corroborated by the fact that between near infrared band $x$ and red band $y$ the correlation may be considerably poorer than that between other spectral bands and the absolute value of the

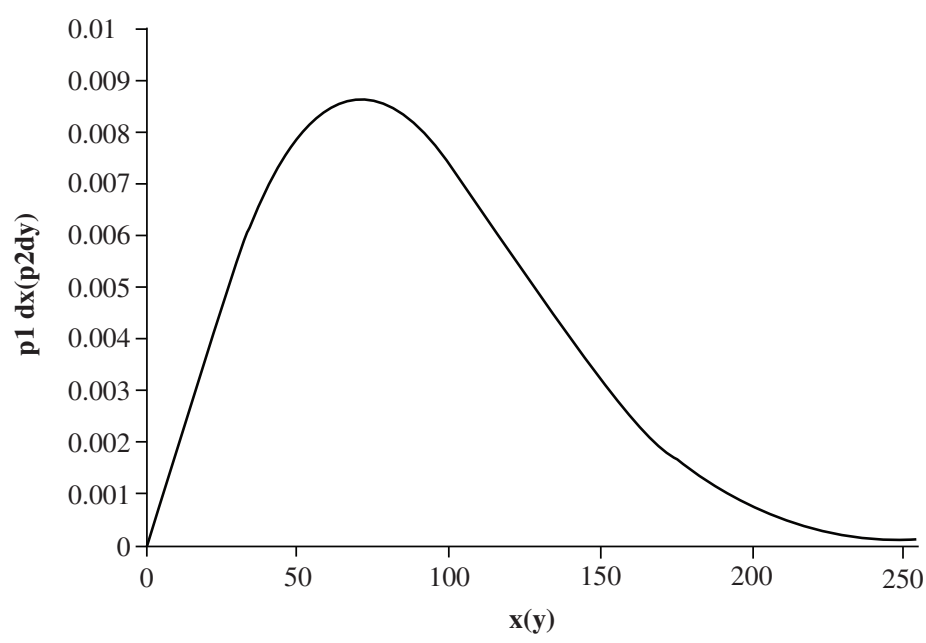

Figure 1: A graphical representation of the distributions $p_{1}$ and $p_{2}$. The $x$ or $y$ values may be represented on the horizontal axis, and the $p_{1}$ or $p_{2}$ values may be represented on the vertical axis. $a_{1}=a_{2}=0.0001$. 
correlation coefficient may be less than 0.5 ([1], pp. 209-212). If there is a strong linear correlation between the infrared and the red band, the relation (6) can not describe reliably the distribution of the $x$ and $y$ brightness values. In such a case, TVI and NDVI are expected to have values which do not differ considerably from pixel to pixel, because of the linear relation between $x$ and $y$. Small differences in the values of the vegetation indices produce image histograms with small deviations. The NIR and Red bands of a satellite image of a broad region, with different land cover types and land uses, are expected to be highly correlated in certain parts of a region and less correlated in other parts. In such a case, the overall correlation between these two bands will not be high and the assumption of the zero correlation may be reasonable.

Based on the bivariate distribution of relation (6) and taking into account well known theorems of statistics, it can be proved [31] that the distribution $g(u)$ of NDVI is given by:

$$
g(u)=\frac{4 \lambda\left(1-u^{2}\right)}{\left[\lambda(1+u)^{2}+(1-u)^{2}\right]^{2}} .
$$

Parameter $\lambda$ is defined to be equal to the ratio $a_{x} / a_{y}$. Since $a_{x}$ and $a_{y}$ are inversely proportional to the square of the standard deviations $\sigma_{x}$ and $\sigma_{y}$ of the brightness values $x$ and $y$, respectively, with the same proportionality constant [31], the following relation may be concluded:

$$
\lambda=\frac{a_{x}}{a_{y}}=\frac{\sigma_{y}^{2}}{\sigma_{x}^{2}} .
$$

From eqns (1) and (3), it can be seen that TVIa is a function of NDVI. More precisely, TVIa may be expressed as:

$$
u\left(u^{\prime}\right)=\sqrt{u^{\prime}}
$$

for a non-negative $u^{\prime} . u$ and $u^{\prime}$ are the values of TVIa and NDVI, respectively.

In the same way, from eqns (2) and (3), it is obvious that TVIb may be expressed in terms of NDVI, as:

$$
u\left(u^{\prime}\right)=\sqrt{u^{\prime}+0.5}
$$

for a non-negative $u^{\prime}+0.5 . u$ and $u^{\prime}$ are the values of TVIa and NDVI, respectively.

According to a well known theorem of statistics [42], the distribution $g(u)$ of $u$ is related with the distribution $g\left(u^{\prime}\right)$ by:

$$
g(u)=g\left[u^{\prime}(u)\right] \cdot\left|\frac{\mathrm{d} u^{\prime}}{\mathrm{d} u}\right|,
$$

where $g\left(u^{\prime}\right)$ is given by eqn (7), where $u$ is replaced by $u^{\prime}$. When the quantities which are under the square roots of eqns (9) and (10) are negative, the respective TVIa and TVIb values are equal to zero, according to eqns (1) and (2). Therefore, the value $g(0)$ of the distribution of TVIa and TVIb is equal to the percentage of the pixels with negative values of $u^{\prime}$ and $u^{\prime}+0.5$, respectively.

In the case of TVIa, $g(0)$ is given by:

$$
g(0)=\int_{-1}^{0} g\left(u^{\prime}\right) \mathrm{d} u^{\prime},
$$

where -1 is the minimum value $u^{\prime}$ of NDVI. 
In the case of TVIb, $g(0)$ is given by:

$$
g(0)=\int_{-1}^{-0.5} g\left(u^{\prime}\right) \mathrm{d} u^{\prime} .
$$

The integration interval -1 to 0.5 is the range at which the quantity $u^{\prime}+0.5$ at the right side of eqn (10) takes negative values.

Combining the eqns (7), (9), (11) and (12), the following relation for the distribution $g(u)$ of TVIa values may be derived:

$$
g(u)= \begin{cases}\frac{8 \lambda\left(1-u^{2}\right) u}{\left(1+u^{2}\right)^{3}\left[\lambda+\left(\frac{1-u^{2}}{1+u^{2}}\right)^{2}\right]^{2}} & (x \geq y), \\ \frac{\lambda}{\lambda+1} & (x<y) .\end{cases}
$$

Combining the eqns (7), (10), (11) and (13), gives the expression for the distribution $g(u)$ of TVIb values, which is:

$$
g(u)= \begin{cases}\frac{8 \lambda\left(1.5-u^{2}\right) u}{\left(0.5+u^{2}\right)^{3}\left[\lambda+\left(\frac{1.5-u^{2}}{0.5+u^{2}}\right)^{2}\right]^{2}} & \left(\frac{x-y}{x+y} \geq-0.5\right), \\ \frac{\lambda}{\lambda+9} & \left(\frac{x-y}{x+y}<-0.5\right) .\end{cases}
$$

In Fig. 2 the distributions of the values of NDVI, TVIa and TVIb are represented, according to the eqns (7), (14) and (15), respectively. The values $u$ have been reduced to the tonality range $[0,1]$. The value of the parameter $\lambda$ is less than unity. It can be observed that particularly the peak of the TVIb distribution is displaced to the right part of the tonality range (high brightness values) and the percentages of small values $u$ are lower than those of the NDVI and TVIa distributions. Therefore, the TVIb image has more pixels with high brightness values than the TVIa and NDVI images. This means that the TVIb image is expected to be brighter than the images of the two other vegetation indices. If $\lambda$ is more than unity, the peak of the TVIb distribution is displaced to the left part of the tonality range (low brightness values).

The standard deviation of the distribution is of particular importance, since it is a measure of the brightness contrast of the image. The standard deviation $\sigma$ of the distribution $g(u)$ is defined by:

$$
\sigma=\sqrt{\int_{R}(u-\mu)^{2} g(u) \mathrm{d} u},
$$

where $\mu$ is the mean value of $g(u)$ and it is defined by:

$$
\mu=\int_{R} u \cdot g(u) \mathrm{d} u .
$$




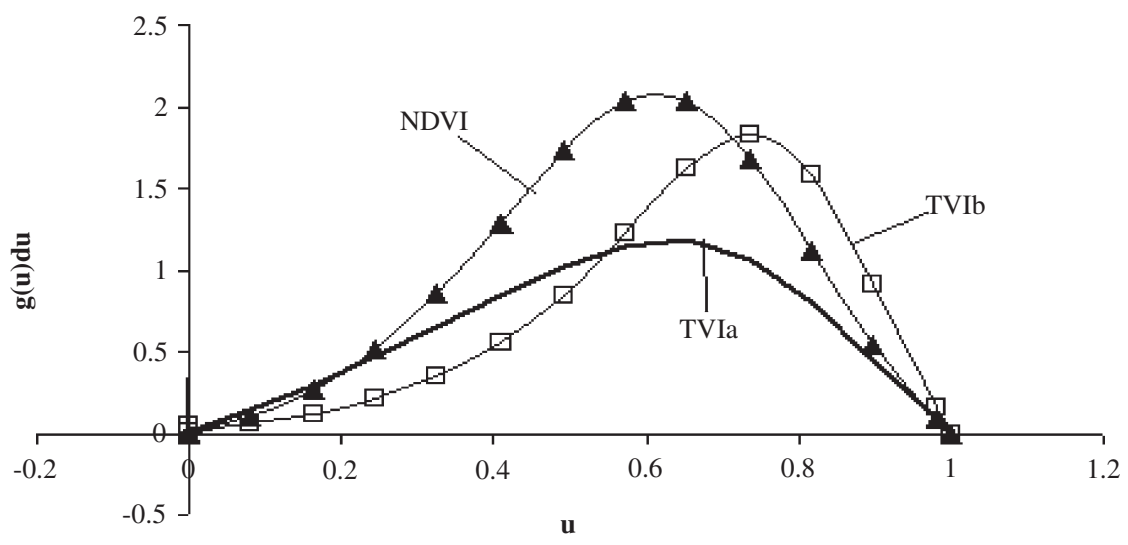

Figure 2: The distributions of the TVIa, TVIb and NDVI values. $\lambda=0.25$.

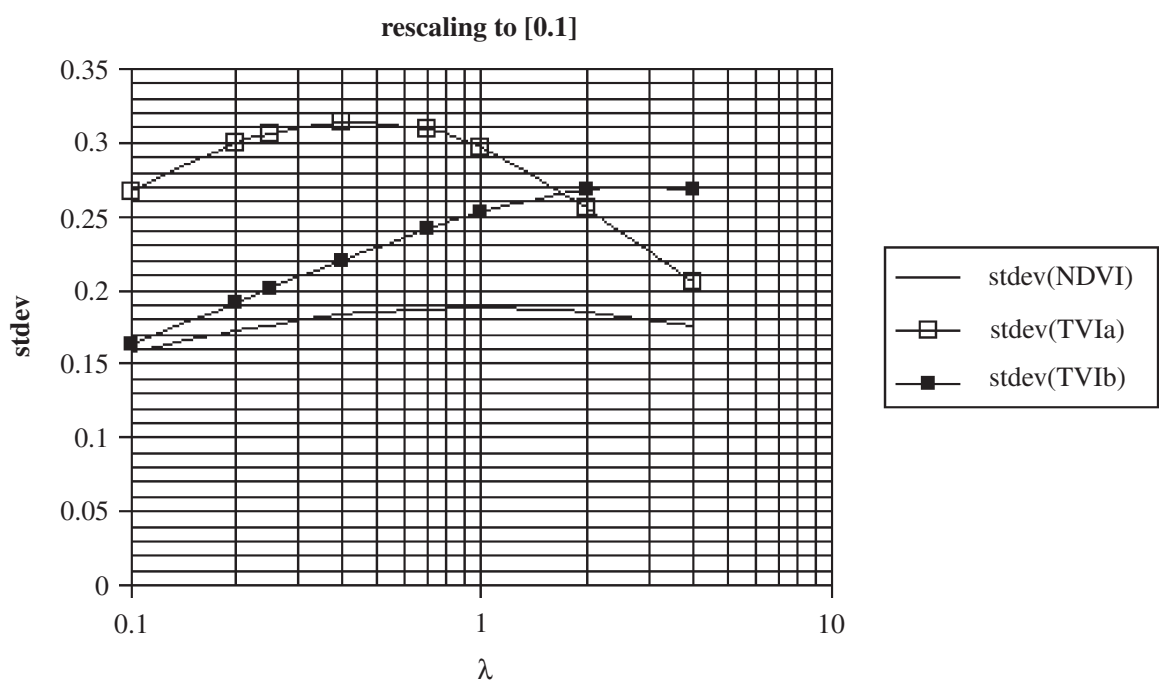

Figure 3: The standard deviations of the TVIa, TVIb and NDVI values against $\lambda$.

$R$ is the integration interval and it is from -1 to 1 for the NDVI distribution, 0 to 1 for the TVIa distribution and 0 to $\sqrt{1.5}$ for the TVIb distribution.

In Fig. 3 the standard deviations of the distributions of the three vegetation indices are presented, against $\lambda$. All values are numerically computed according to eqns (16) and (17) and reduced to the tonality range $[0,1]$. The function $g(u)$ is given by eqns (7), (14) and (15), in order to calculate the standard deviation of the NDVI, the TVIa and the TVIb distributions, respectively.

It can be observed that the standard deviation of the TVIa distribution is considerably bigger than those of the TVIb and NDVI distributions, when $\lambda$ does not exceed unity. This means that the TVIa image is expected to have a better brightness contrast than that of the two other vegetation indices. The standard deviations of TVIb and NDVI differ very little when $\lambda$ is a few times less than unity. As long as $\lambda$ increases, the standard deviation of TVIb becomes increasingly higher than that of NDVI and it is the highest of the three vegetation indices, when $\lambda$ is considerably more than unity. 
The standard deviation is a measure of the brightness contrast of the image. A study of the signal to noise ratio of the three vegetation indices may give more insight about their sensitivity over targets of different tonalities.

\section{A STUDY OF THE SIGNAL TO NOISE RATIO}

The signal to noise ratio of the image of a vegetation index is a measure of how well the vegetation index responds over regions with a different vegetation cover, in the presence of signals which are irrelevant to the targets of interest (noise). In this paper, the $x$ (infrared) and $y$ (red) brightness values are considered to contain a Gaussian noise with a constant standard deviation $\sigma_{\mathrm{n}}$. This standard error represents the differences in the tonality between pixels, which belong to the same land cover type but, despite this, they do not have exactly the same spectral signature. This assumption may help in deducing analytical expressions and making predictions about the signal to noise ratio, which can be tested by real data.

There are several ways to define the signal to noise ratio. In the present paper, the following expression is employed [40]:

$$
S N R=\frac{\sigma}{\sigma_{u}}
$$

SNR is the signal to noise ratio of the image of a vegetation index $u$. $u$ is the function of $x$ and $y$, which defines the vegetation index, according to eqns (1), (2) or (3). $\sigma_{u}$ is the standard deviation of a $u$ value of a certain pixel, which depends on the standard deviations of $x$ and $y$ values of this pixel and generally it is not constant for every $u . \sigma$ is the standard deviation of the tonality distribution $g(u)$ and it is defined according to relation (16).

The parameter $\sigma$ represents the range of values of the vegetation index in the whole image. As long as $\sigma$ increases, the differences in the value of the vegetation index between different regions of different land cover types are expected to increase. This favors a 'good' signal, in the sense that if the differences in the vegetation index are big the different land cover types may be expressed more clearly.

The parameter $\sigma_{u}$ expresses the range of the local variations in the value of the vegetation index, which may occur between pixels of the same region and same land cover type. These local variations occur because pixels of the same land cover type do not necessarily have exactly the same NIR and Red tonalities and, consequently, they may have different vegetation index values. As long as $\sigma_{u}$ increases, it does not favor the recognition of different land cover types.

Taking into account the physical meaning of the parameters that appear in the right part of eqn (18), $\sigma$ and $\sigma_{u}$ may be considered as the 'signal' and the 'noise' of the image, respectively.

It can be proved (see Appendix) that the signal to noise ratio SNR(TVIa) of the image of TVIa is given by:

$$
\operatorname{SNR}(\mathrm{TVIa})=\frac{\sigma(\lambda) \cdot y}{\sigma_{n}} \cdot \sqrt{\frac{r-1}{(r+1)\left(r^{2}+1\right)}} \cdot(r+1)^{2} \quad \text { for } \quad r \geq 1,
$$

where $\sigma_{n}$ is the standard deviation of the $x$ and $y$ values of a pixel (see Appendix).

$r$ is defined as:

$$
r=\frac{x}{y}
$$

The parameter $\lambda$ in the parenthesis expresses the dependency of the standard deviation $\sigma$ of the vegetation index on $\lambda$. 
If $x<y$, the signal to noise ratio cannot be calculated according to eqn (18) and it is defined to be zero.

$$
S N R \text { (TVIa) }=0 \text { for } \quad r<1 .
$$

The physical meaning of eqn (21) is that for $x<y$, since TVI is equal to zero (according to eqn (1)), it is not sensitive to changes in $x$ and $y$ values.

In a similar way, we derived the expression for the signal to noise ratio $S N R$ (TVIb) of TVIb, which is given by:

$$
\begin{gathered}
S N R(\mathrm{TVIb})=\frac{\sigma(\lambda) \cdot y}{\sigma_{n}} \cdot \sqrt{\frac{\frac{r-1}{r+1}+0.5}{r^{2}+1}} \cdot(r+1)^{2} \quad \text { for } \quad r \geq 1 / 3, \\
S N R(\mathrm{TVIb})=0 \text { for } \quad r<1 / 3 .
\end{gathered}
$$

It can also be proved that the expression for the signal to noise ratio $S N R$ (NDVI) of NDVI is given by:

$$
S N R(\mathrm{NDVI})=\frac{\sigma(\lambda) \cdot y}{2 \sigma_{n}} \cdot \frac{(r+1)^{2}}{\sqrt{r^{2}+1}} .
$$

In Fig. 4, the signal to noise ratios of the three vegetation indices against $\lambda$ are presented, for $y / \sigma_{n}$ equal to unity. The curves have been calculated according to eqns (19), (22) and (24). The standard deviation $\sigma$ has been calculated numerically according to eqn (16). It can be observed that the signal to noise ratios of TVIa and TVIb are higher than that of NDVI, for $\lambda<2$. For $\lambda>2$, the signal to noise ratio of TVIa is less than that of NDVI and the signal to noise ratio of TVIb is the biggest.

In Fig. 5, the signal to noise ratios of TVIa, TVIb and NDVI against $r$ are presented, for $\lambda$ and $y / \sigma_{n}$ equal to unity. For relatively high values of $r$ (more than unity), the signal to noise ratio of TVIa and TVIb is better than that of NDVI. NDVI has a better signal to noise ratio than that of the two other indices for low values of $r$.

It is interesting to study the behavior of the ratios $S N R$ (TVIa)/SNR(NDVI) and $S N R(\mathrm{TVIb}) /$ $S N R$ (NDVI). Combining eqns (19), (21) and (24) gives:

$$
\frac{S N R(\mathrm{TVIa})}{S N R(\mathrm{NDVI})}=\frac{2 \sigma_{\mathrm{TVIa}}(\lambda)}{\sigma_{\mathrm{NDVI}}(\lambda)} \cdot \sqrt{\frac{r-1}{r+1}} \text { for } \quad r \geq 1,
$$

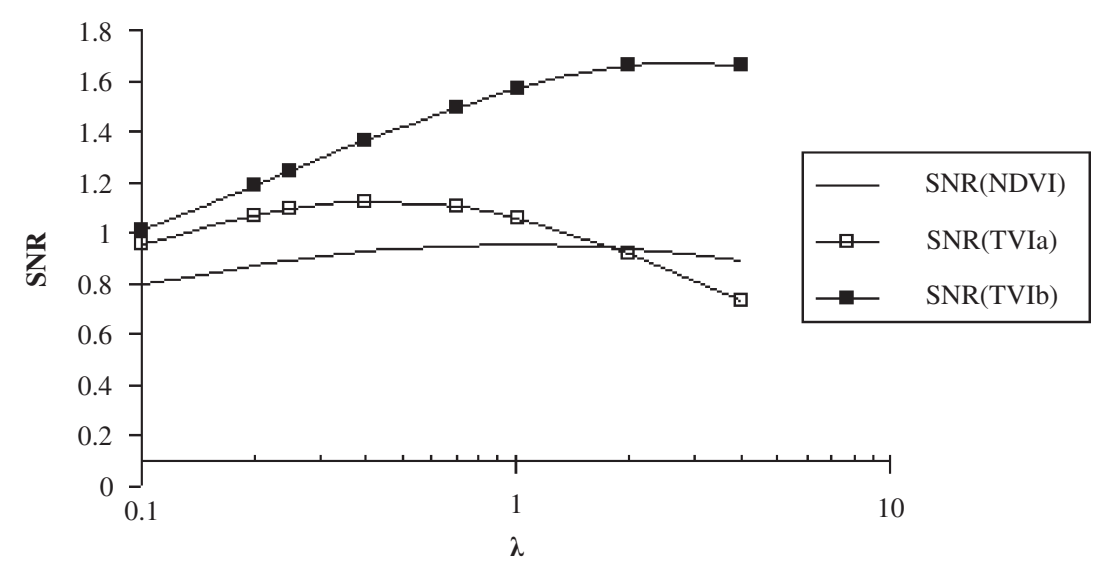

Figure 4: The signal to noise ratio of the TVIa, TVIb and NDVI values against $\lambda . y / \sigma_{n}=1, r=3$. 


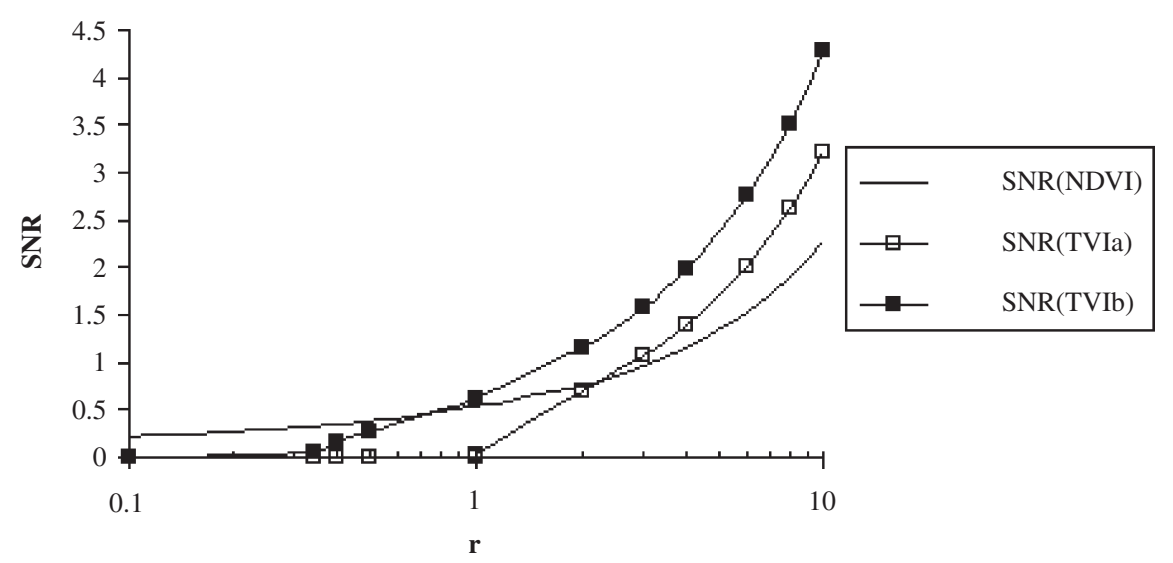

Figure 5: Signal to noise ratios of TVIa, TVIb and NDVI against $r . y / \sigma_{n}=1, \lambda=1$.

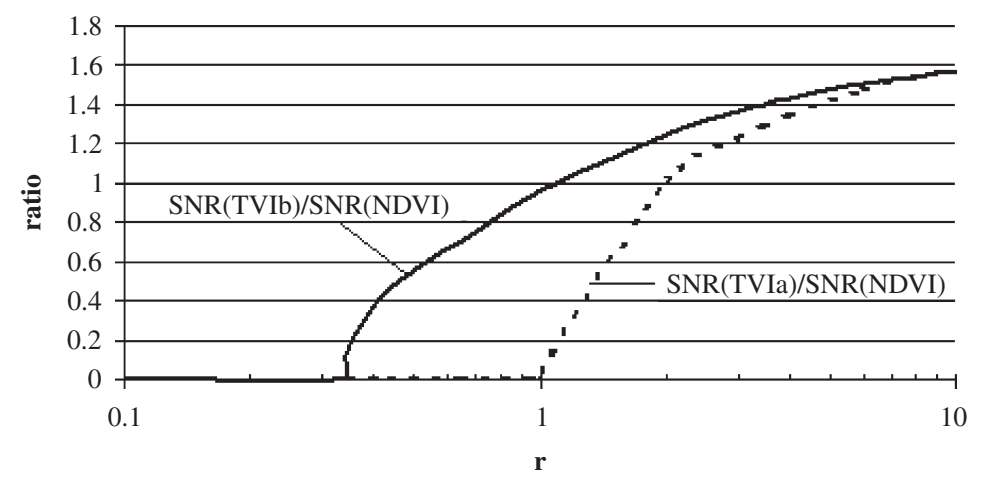

Figure 6: A graphical representation of the ratios $S N R$ (TVIa)/SNR(NDVI) and $S N R$ (TVIb)/SNR (NDVI) against $r \cdot \lambda=0.217$.

$$
\frac{S N R \text { (TVIa) }}{S N R \text { (NDVI) }}=0 \quad \text { for } \quad r<1 .
$$

$\sigma_{\mathrm{TVIa}}$ and $\sigma_{\mathrm{NDVI}}$ are the standard deviations of the distributions of TVI and NDVI values, respectively, and they depend on $\lambda$.

Combining eqns (22), (23) and (24) gives:

$$
\begin{gathered}
\frac{S N R(\mathrm{TVIb})}{S N R(\mathrm{NDVI})}=\frac{2 \sigma_{\mathrm{TVIb}}(\lambda)}{\sigma_{\mathrm{NDVI}}(\lambda)} \cdot \sqrt{\frac{r-1}{r+1}} \text { for } r \geq 1 / 3, \\
\frac{S N R(\mathrm{TVIb})}{S N R(\mathrm{NDVI})}=0 \text { for } \quad r<1 / 3 .
\end{gathered}
$$

$\sigma_{\text {TVIb }}$ is the standard deviation of the distribution of the TVIb values.

In Fig. 6, the ratios $S N R$ (TVIa) $/ S N R$ (NDVI) and $S N R$ (TVIb)/SNR(NDVI) against $r$ are presented, for $\lambda$ less than unity. It can be observed that for $r>2$, both ratios are more than unity, which means that the signal to noise ratios of TVIa and TVIb are better than that of NDVI. The ratio $S N R$ (TVIb)/SNR(NDVI) is always more than $S N R$ (TVIa)/SNR(NDVI), but at high $r$ values these two 
ratios tend to equalize. This means that the signal to noise ratio of TVIb is better than that of TVIa but at high $r$ values the signal to noise ratios do not differ considerably.

For $\lambda$ more than unity, we have found that the signal to noise ratio of TVIb is considerably better than that of TVIa and NDVI, even for high $r$ values.

The general conclusion of the study of the signal to noise ratio is that TVIa and TVIb have a better $S N R$ than that of NDVI, for ratio $r$ more than unity. The theoretical predictions about the standard deviations and the signal to noise ratios of the three vegetation indices have to be tested with real data.

\section{EXPERIMENTATION WITH SATELLITE IMAGES}

In order to check the theoretical predictions about the behavior of the three vegetation indices, we used two satellite images with a considerably different spatial resolution: a Landsat 7 ETM image of Zakynthos island (western Greece), which was taken in August 1999 and a SPOT vegetation image of the Mediterranean Sea (September 2000). The Landsat image has a $30 \mathrm{~m}$ spatial resolution in the NIR and Red bands. The SPOT vegetation image has a $1 \mathrm{~km}$ spatial resolution. The experimentation with the two images was focused on how the three vegetation indices respond over regions with land covers of a small extension, such as the burnt areas of Zakynthos, as well as over structures of a much larger scale, such as Nile river zone (Egypt). The response of TVI over burnt areas is of particular importance, since vegetation indices are used to map burnt areas of Greek territory [43-45]. Quantitative comparisons between the theoretically predicted and actual standard deviations of the images of the vegetation images were also made. The effect of the atmospheric scattering has been removed from the first image, applying the technique of relative atmospheric correction [46]. In the second image, there is a zero offset from the beginning of the axes of the histogram of the Red and the NIR channels, therefore there is no meaning to apply this technique.

The behavior of the image of Zakynthos is first studied. A fire had taken place shortly before the acquisition of the image. There is a burnt area at the center of the island, which appears with dark tones in Figs 7, 8, 10, 11, 13 and 14. The standard deviation $\sigma_{x}$ of the NIR channel is equal to 34.458 and the standard deviation $\sigma_{y}$ of the Red channel is 16.038 .

According to eqn (8), the value of $\lambda$ is:

$$
\lambda=\frac{\sigma_{y}^{2}}{\sigma_{x}^{2}}=0.217 .
$$

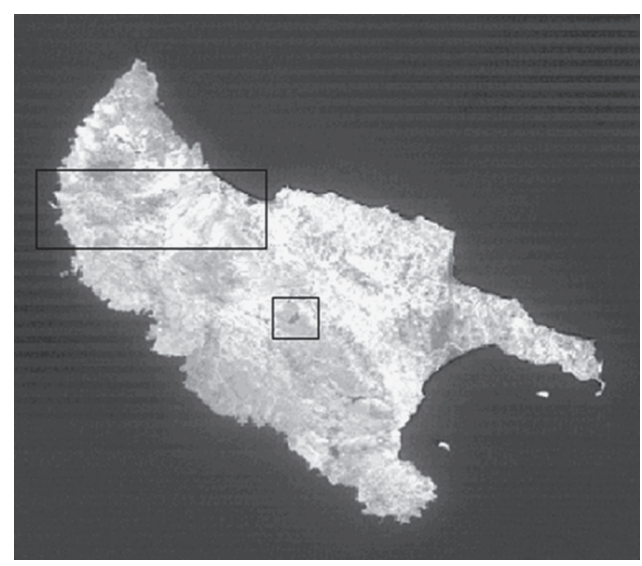

Figure 7: The NDVI image of the island of Zakynthos. 


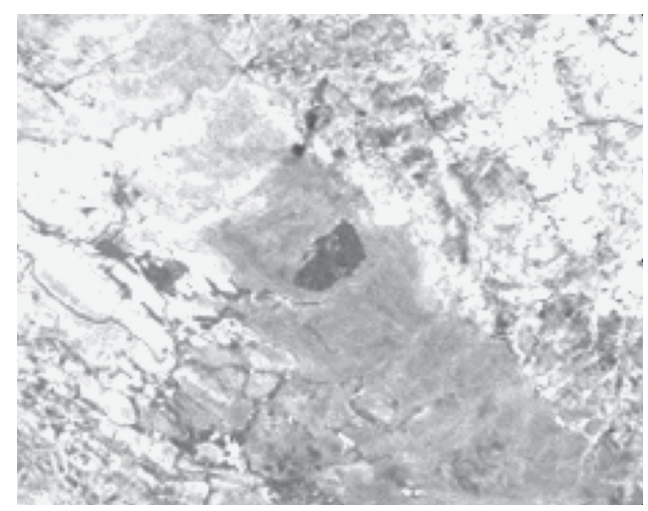

Figure 8: The burnt area at the center of the NDVI image.

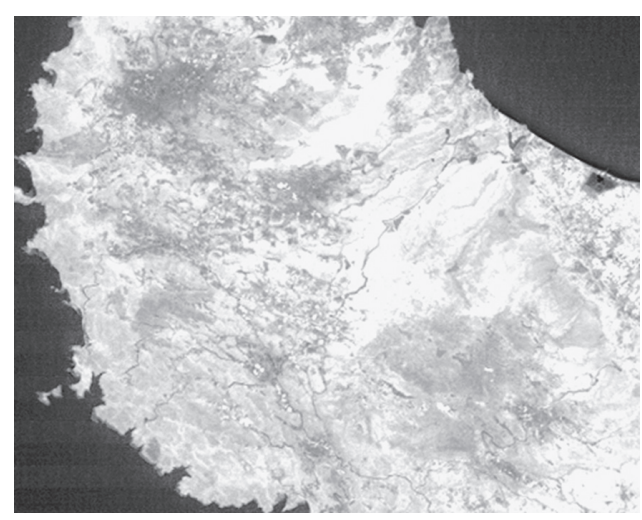

Figure 9: The northern part of the NDVI image.

In Figs 7, 10 and 13, we can see the images of NDVI, TVIb and TVIa, respectively, over the whole island. The small rectangle at the center is surrounding the burnt area, which is zoomed out in Figs 8, 11 and 14. The burnt area was identified by in situ observations and information given by the municipalities of the island. The location of the burnt area in the satellite images coincides with that of field data. The larger rectangle at the north of the island (Figs 7, 10 and 13) is zoomed out in Figs 9, 12 and 15. In these images, we can see curved lineaments, which represent the road network of the area.

It can be seen that in the images of Figs 7 and 10 there is a diffuse luminance, especially in the sea around the island, where parallel zones of diverse tonality appear. This luminance does not appear in the TVIa image of Fig. 13 and the sea is expressed with dark tones. On the other hand, in the zoomed TVIa images of Figs 14 and 15, the burnt areas, as well as the road network, appear more clearly, with dark tones. In another Landsat image of Western Peloponnese, TVIa was successful in mapping the drainage network of a basin.

In Table 1 the theoretically predicted and the actual standard deviation values (stdev) of the images of the three vegetation indices are presented. The theoretical estimations were made using the curves of Fig. 4 , for $\lambda$ equal to 0.22 (according to eqn (29)). The actual values were computed using the ERDAS 


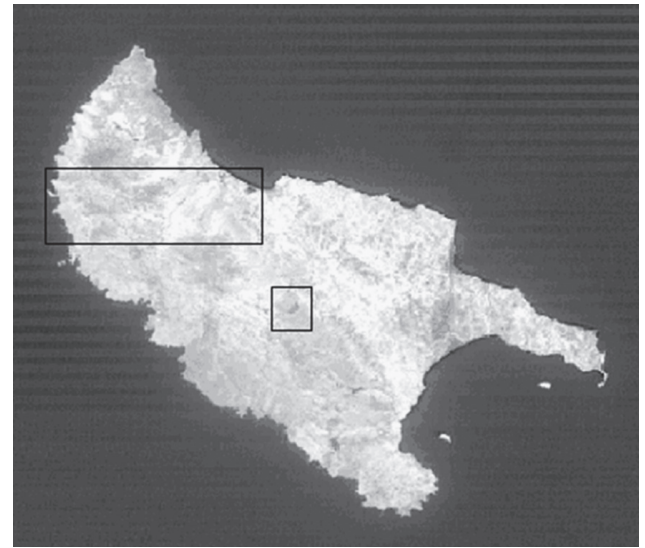

Figure 10: The TVIb image of Zakynthos island.

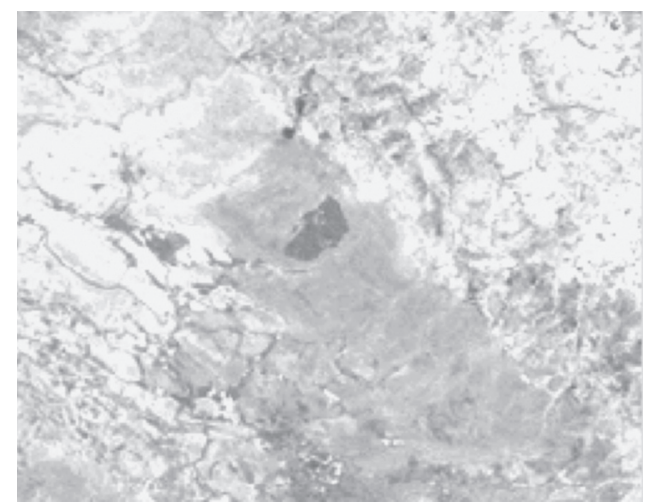

Figure 11: The burnt area at the center of the TVIb image.

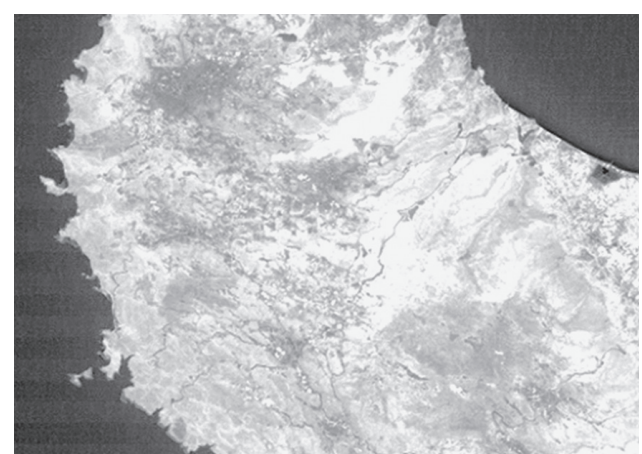

Figure 12: The northern part of the TVIb image.

Imagine 8.6 software package and reduced to the $[0,1]$ range. We can see that the actual standard deviation of the TVIa image is considerably bigger than that of the two other vegetation indices, in accordance with the theoretical predictions. On the other hand, the actual standard deviations of the NDVI and the TVIb images are considerably smaller than the theoretically predicted values. 


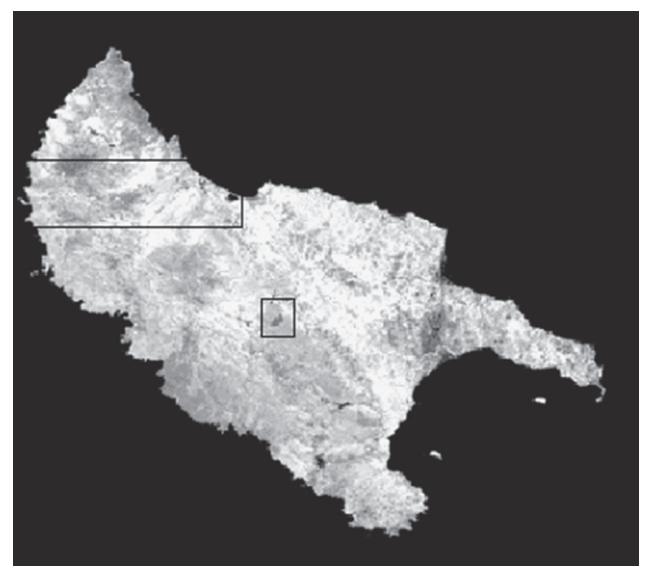

Figure 13: The TVIa image of Zakynthos island.

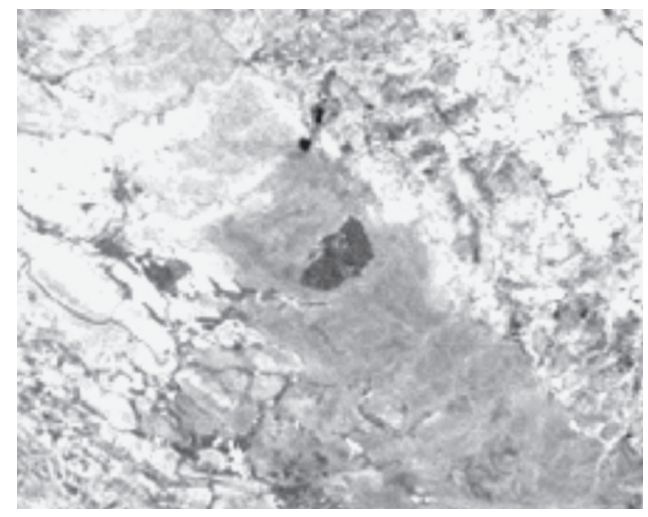

Figure 14: The burnt area at the center of the TVIa image.

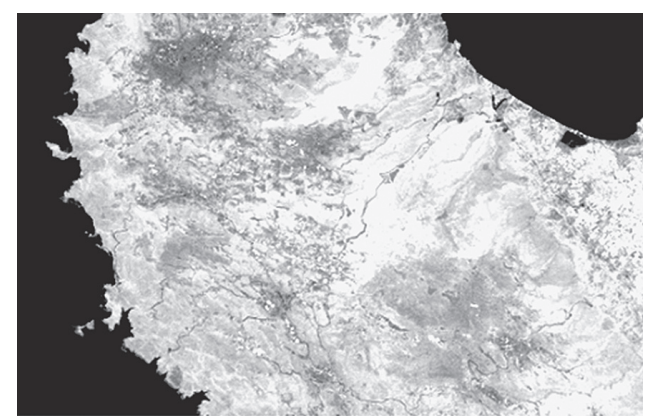

Figure 15: The northern part of the TVIa image.

In Figs 16, 17 and 18 the NDVI, the TVIb and the TVIa images of the region of the south east Mediterranean Sea are presented, respectively. This is part of the SPOT image. We can see the zone of the Nile River in Egypt, as well as its delta. In all images the river zone and the delta are presented with very bright tones, because there is considerable vegetation around the river and an arid region 
Table 1: Comparison of theoretical predictions and actual statistical values of the Landsat 7 image of Zakynthos island.

\begin{tabular}{lccc}
\hline Vegetation index & NDVI & TVIb & TVIa \\
\hline stdev actual & 0.096 & 0.098 & 0.261 \\
stdev theoretical & 0.173 & 0.193 & 0.301 \\
\hline
\end{tabular}

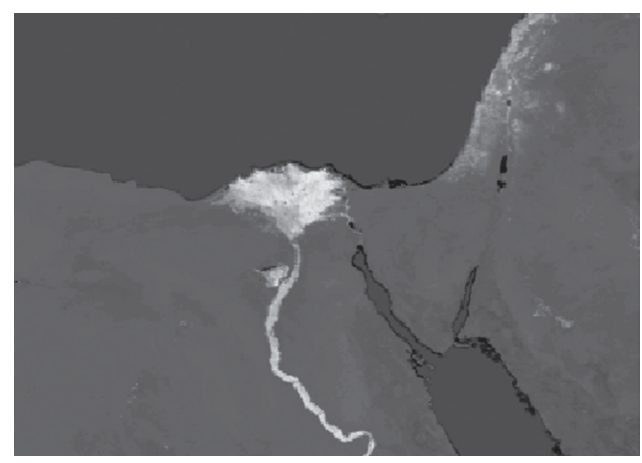

Figure 16: The NDVI image of Egypt.

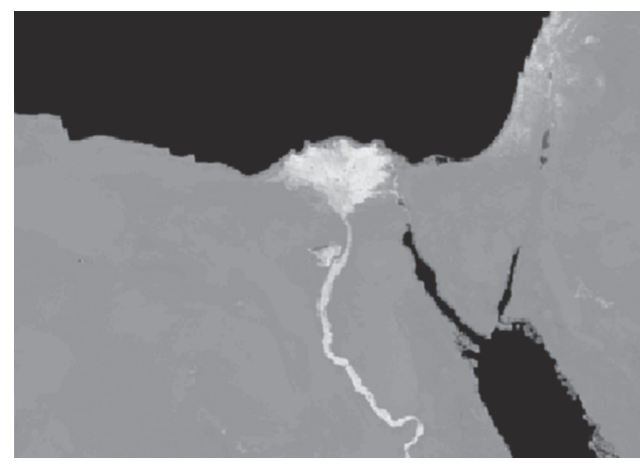

Figure 17: The TVIb image of Egypt.

out of the river zone. In the TVIb image, the arid region is expressed in bright tones and the contrast between the river zone and the surrounding region is less than that of the other two images.

In Table 2 the standard deviations of the vegetation indices of the SPOT image are presented. There is a generally good agreement between theoretically predicted and actual values. The highest percentage deviation between theoretical and actual standard deviation is recorded in the case of NDVI $(25 \%)$ and the smallest deviation is recorded in the case of TVIa (5.5\%). In all tables, there is an agreement between theoretical and actual values, in terms of comparing standard deviations of different vegetation indices. For example, in Table 2, the standard deviation of the NDVI image is more than that of the TVIb image, according to theoretical prediction and actual standard deviations. 


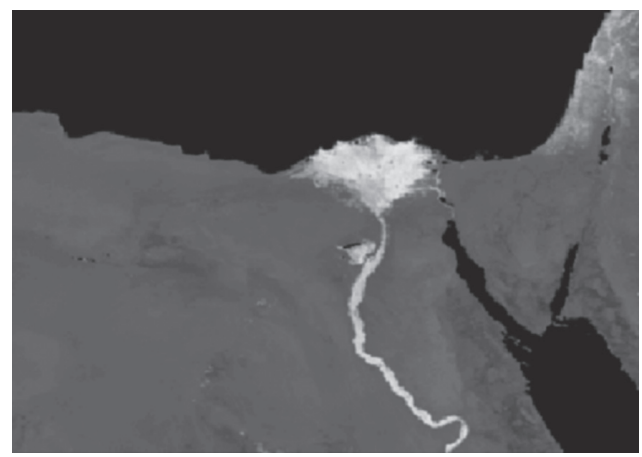

Figure 18: The TVIa image of Egypt.

Table 2: Comparison of theoretical predictions and actual statistical values of the SPOT image of the region of Eastern Mediterranean Sea.

\begin{tabular}{llll}
\hline Vegetation index & NDVI & TVIb & TVIa \\
\hline stdev actual & 0.134 & 0.282 & 0.325 \\
stdev theoretical & 0.178 & 0.240 & 0.308 \\
\hline
\end{tabular}

We should expect deviations between the theoretically predicted and the calculated image stdev values, since the distributions $p_{1}, p_{2}$ and $f$, upon which we have elaborated the whole probabilistic approach, are only rough approximations of real image histograms. The reliability of the mathematical analysis which we have developed in this paper may be evaluated by comparing the behavior of the theoretically predicted values with that of the actual image values. According to this criterion, we think that the probabilistic approach works satisfactorily in estimating the statistical performance of the vegetation indices, since it can predict whether the standard deviation of a vegetation index is bigger or smaller than that of the other one.

The signal to noise ratio of the NDVI, TVIa and TVIb images of the Landsat scene was then calculated, for each pixel. This was done according to eqn (18). In the numerator we put the actual standard deviation of the image of the vegetation index. In the denominator we put the standard deviation of a $3 \times 3$ window, in the central pixel of which we attributed the calculated $S N R$ value. In this way, we produced the $S N R$ (NDVI), the $S N R$ (TVIa) and the $S N R$ (TVIb) images of NDVI, TVIa and TVIb, respectively. Then, we produced the images of the ratios $S N R$ (TVIa)/SNR(NDVI) and $S N R(\mathrm{TVIb}) / S N R(\mathrm{NDVI})$. The necessary calculations were made using the 'spatial modeler' and other utilities of the ERDAS Imagine 8.6 software package.

Finally, we produced the image of the ratio $r$ equal to NIR/Red and we selected 50 check points, scattered at the whole image. For each check point, we recorded the values of $r, S N R$ (TVIa)/SNR (NDVI) and $S N R$ (TVIb)/SNR(NDVI). In Fig. 19, the experimental $S N R$ (TVIa)/SNR(NDVI) and $S N R$ (TVIb)/SNR(NDVI) values against $r$, are presented. It is interesting to compare the graphs of Figs 19 and 6, since the theoretical curves of Fig. 6 were computed for the same value of the parameter $\lambda$. It can be seen that in both figures the ratio $S N R(\mathrm{TVIb}) / S N R(\mathrm{NDVI})$ is bigger than the ratio $S N R$ (TVIa)/SNR(NDVI) for relatively low $r$ values. Theoretical predictions and real data show that the two ratios are less than unity for $r$ less than 2 and more than unity for higher $r$ values. As long as $r$ increases, the difference between the two ratios decreases. Theoretically, this difference tends 


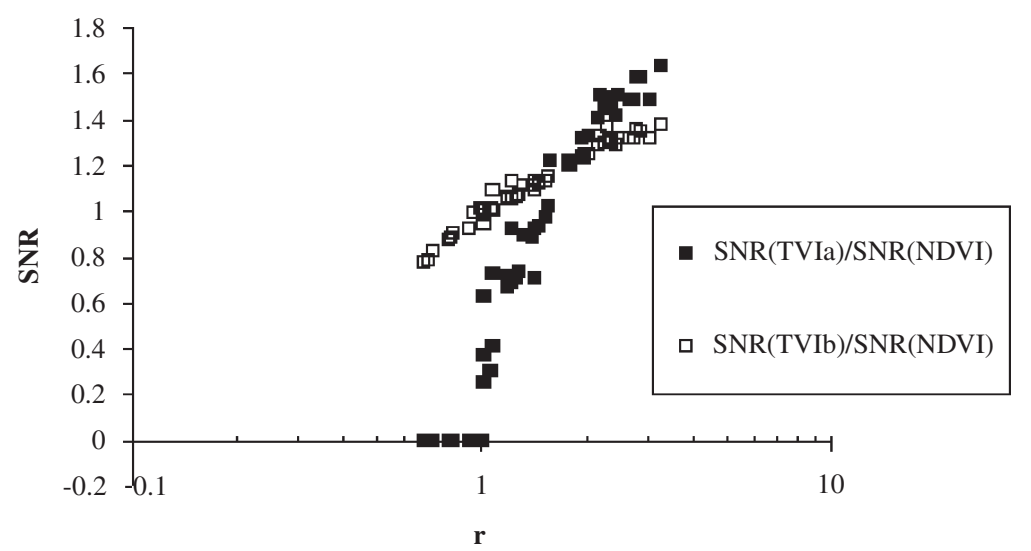

Figure 19: A graphical representation of the experimental values of the ratios $S N R$ (TVIa)/SNR(NDVI) and $S N R(\mathrm{TVIb}) / S N R(\mathrm{NDVI})$ against $r$.

asymptotically to zero. The experimental results showed that the two curves coincide at about $r=2$. For $r>2$, the $S N R$ (TVIa)/SNR(NDVI) values become higher than the $S N R(\mathrm{TVIb}) / S N R$ (NDVI) values. The physical meaning of these graphs is that when the brightness value at the NIR zone is not much higher than the respective brightness value at the Red zone, the signal to noise ratio of NDVI is better than that of TVIa and TVIb. In regions with a dense vegetation cover, where the NIR brightness value is quite higher than that of the Red zone, the signal to noise ratio of the TVIa and the TVIb images is better than that of NDVI. The TVIb image has a better signal to noise ratio than the TVIa image for low $r$ values, but this difference tends to be eliminated, or even reversed, at higher $r$ values.

\section{CONCLUSIONS}

According to the mathematical analysis and the experimentation with the satellite image, the following conclusions may be drawn.

The standard deviation of the TVIa image is considerably higher than that of the TVIb and the NDVI images, if the standard deviation of the NIR channel is not smaller than the standard deviation of the Red channel. In such a case, the application of TVIa produces images with a good brightness contrast.

The signal to noise ratio of a vegetation index depends on the standard deviations of the NIR and the Red channel, as well as the ratio $r$ of the NIR brightness value to the Red brightness value. For small $r$ values the signal to noise ratio of the NDVI image is better than that of TVIa and TVIb. As long as $r$ increases, the signal to noise ratio of the TVIa and the TVIb images is better than that of the NDVI image.

The mathematical analysis has further shown that as long as the ratio of the standard deviation of the NIR channel to the standard deviation of the Red channel increases, the signal to noise ratio of the TVIb image gets bigger than the signal to noise ratio of the TVIa image, for high $r$ values. Therefore, TVIb is more efficient in mapping and recognizing areas with dense vegetation cover (high $r$ values). TVIa produces images with a good contrast, in such a way that regions with a very sparse vegetation cover, or burnt areas, appear in dark tones and can be easily distinguished from the surrounding formations. In satellite images with a relatively good spatial resolution (for example Landsat images with $15 \mathrm{~m}$ or $30 \mathrm{~m}$ spatial resolution), the road network or the drainage network of a river can be quite clearly presented in a TVIa image, with dark tones. In satellite images with poor 
spatial resolution, like SPOT vegetation images (which have a $1 \mathrm{~km}$ spatial resolution), river zones and deltas may be expressed with bright tones in TVIa and NDVI images, if the vegetation cover is considerably dense compared to that of the surrounding formations. The probabilistic approach that is developed in this paper may be also used in assessing the efficiency, or modifying, other vegetation indices, which are employed in environmental research.

\section{APPENDIX: DERIVATION OF EQN (19)}

The quantity $\sigma_{u}$ is calculated by Spiegel [42]:

$$
\sigma_{u}=\sqrt{\left(\frac{\partial u}{\partial x} \sigma_{x}\right)^{2}+\left(\frac{\partial u}{\partial y} \sigma_{y}\right)^{2}} .
$$

$\sigma_{x}$ and $\sigma_{y}$ are the standard deviations of $x$ and $y$, respectively. $u$ is the function of $x$ and $y$ by which the vegetation index is calculated.

The standard deviations of brightness values $x$ and $y$ are assumed to be constant and equal to $\sigma_{n}$, according to the equation:

$$
\sigma_{x}=\sigma_{y}=\sigma_{n} .
$$

Combining (A.1) and (A.2) gives:

$$
\sigma_{u}=\sigma_{n} \sqrt{\left(\frac{\partial u}{\partial x}\right)^{2}+\left(\frac{\partial u}{\partial y}\right)^{2}} .
$$

Combining eqns (1) and (A.3), we can find the expression for $\sigma_{u}$ of TVIa when $x \geq y$ :

$$
\sigma_{u}=\frac{\sigma_{n}}{y} \cdot \sqrt{\frac{(r+1) \cdot\left(r^{2}+1\right)}{r-1}} \cdot \frac{1}{(r+1)^{2}},
$$

where $r$ is defined by eqn (20)

Combining eqns (1) and (A.3), it can be easily proved that when $x<y, \sigma_{u}$ of TVIa is:

$$
\sigma_{u}=0 .
$$

Combining (18) and (A.5) gives the signal to noise ratio SNR(TVIa) of TVIa, which is:

$$
S N R(\text { TVIa })=\frac{\sigma(\lambda) \cdot y}{\sigma_{n}} \cdot \sqrt{\frac{r-1}{(r+1) \cdot\left(r^{2}+1\right)}} \cdot(r+1)^{2} \quad \text { for } \quad x \geq y,
$$

For $x<y$ the standard deviation $\sigma_{u}$ is equal to zero, according to eqn (A.5); therefore, the signal to noise ratio of TVIa can not be calculated according to eqn (18).

\section{REFERENCES}

[1] Jensen, R.J., Introductory Digital Image Processing: A Remote Sensing Perspective. Prentice Hall, 1996.

[2] Rouse, J.W., Haas, R.H., Schell, J.A. \& Deering, D.W., Monitoring vegetation systems in the Great Plains with ERTS. 3rd ERTS Symposium, Vol. 1, pp. 48-62, 1973.

[3] Deering, D.W., Rouse, J.W., Haas, R.H. \& Schell, J.A., Measuring forage production of grazing units from Landsat MSS Data. 10th International Symposium on Remote Sensing of Environment, Vol. 2, pp. 1169-1178, 1975. 
[4] Huete, A.R., A soil-adjusted vegetation index (SAVI). Remote Sensing of Environment, 25, pp. 295-309, 1988.

[5] Faust, N.L., Image enhancement. Encyclopedia of Computer Science and Technology, Vol. 20, Supplement 5, eds A. Kent \& J.G. Williams, Marcel Dekker Inc., 1989.

[6] Baret, F., Guyot, G. \& Major, D.J., TSAVI: a vegetation index which minimizes soil brightness effects on LAI and APAR estimation. Proc. IGARSS'89 and 12th Canadian Symposium on Remote Sensing. Vancouver, Canada, pp. 1355-1358, 1989.

[7] Baret, F. \& Guyot, G., Potentials and limits of vegetation indices for LAI and APAR assessment. Remote Sensing of Environment 35, pp. 161-173, 1991.

[8] Qi, J., Chehbouni, A., Huete, A.R., Kerr, Y.H. \& Sorooshian, S., A modified soil adjusted vegetation index. Remote Sensing of Environment, 48(2), pp. 119-126, 1994.

[9] Burgan, R.E., Use of remotely sensed data for fire danger estimation. Earsel advances in remote sensing. Remote Sensing and GIS applications for Forest Fire Management, 4(4), pp. 1-8, 1996.

[10] Rondeaux, G., Steven, M. \& Baret, F., Optimization of soil-adjusted vegetation indices. Remote Sensing of Environment, 55, pp. 95-107, 1996.

[11] Chen, J. \& Cihlar, J., Retrieving leaf area index of boreal conifer forests using Landsat Thematic Mapper. Remote Sensing of Environment, 55, pp. 153-162, 1996.

[12] Fassnacht, K.S., Gower, S.T., Mackenzie, M.D., Nordheim, E.V. \& Lillesand, T.M., Estimating the leaf area index of north central Wisconsin forest using Landsat Thematic Mapper. Remote Sensing of Environment, 61, pp. 229-245, 1997.

[13] Spanner, M.A., Pierce, L.L., Peterson, D.L. \& Running, S.W., Remote sensing of temperate coniferous forest leaf area index. The influence of canopy closure understory vegetation and background reflectance. International Journal of Remote Sensing, 11, pp. 95-111, 1990.

[14] Brown, L., Jin, M.C., Lablanc, S.G. \& Cihlar, J., A shortwave infrared modification to the simple ratio for LAI retrieval in boreal forests: An image and model analysis. Remote Sensing of Environment, 71, pp. 16-25, 2000.

[15] Chen, J., Evaluation of vegetation indices and modified simple ratio for boreal applications. Can. J. Remote Sens., 22, pp. 229-242, 1996.

[16] Nemani, R., Pierce, L., Running, S. \& Band, L., Forest ecosystem processes at the watershed scale: sensitivity to remotely sensed leaf area index estimates. International Journal of Remote Sensing, 14, pp. 2519-2534, 1993.

[17] Rahman, M.M., Csaplovics, E. \& Koch, B., An efficient regression strategy for extracting forest biomass information from satellite sensor data. International Journal of Remote Sensing, 26(7), pp. 1511-1519, 2005.

[18] Chaurasia, S. \& Dakwal, V. K., Comparison of principal component inversion with VI-empirical approach for LAI estimation using simulated reflectance data. International Journal of Remote Sensing, 25(14), pp. 2881-2887, 2004.

[19] Mutanga, O. \& Skidmore, A.K., Narrow band vegetation indices overcome the saturation problem in biomass estimation. International Journal of Remote Sensing, 25(19), pp. 3999-4014, 2004.

[20] Murphy, R.J., Tolhurst, T.J., Chapman, M.G. \& Underwood, A.J., Estimation of surface chlorophyll-a on an emersed mudflat using field spectrometry: accuracy of ratios and derivative-based approaches. International Journal of Remote Sensing, 26(9), pp. 1835-1859, 2004.

[21] Gitelson, A.A., Wide dynamic range vegetation index for remote quantification of biophysical characteristics of vegetation. Journal of Plant Physiology, 161, pp. 165-173, 2004.

[22] Mallinis, G., Koutsias, N., Makras, A. \& Karteris, M., An assessment of the information content of Landsat $-5 \mathrm{TM}$ bands for the estimation of forest stand parameters in Kassandra peninsula. 
Proceedings of the 7th Panhellenic Geographical Congress, Mytilini (Greece), October 14-17, Vol. II, pp. 415-422, 2004.

[23] Kale, M., Singh, S. \& Roy, P.S., Estimation of leaf area index in dry deciduous forests from IRS-WiFS in Central India. International Journal of Remote Sensing, 26(21), pp. 4855-4867, 2005.

[24] Silleos, N.G., Alexandridis, T.K., Gitas, I.Z. \& Perakis, K., Advances made in biomass estimation and vegetation monitoring in the last 30 years. Geocarto International, 21(4), pp. 21-28, 2006.

[25] Royo, C., Aparicio, N., Villegas, D., Casadesus, J., Monneveu, P. \& Araus, J.L., Usefulness of spectral reflectance indices as durum wheat yield predictors under contrasting Mediterranean conditions. International Journal of Remote Sensing, 24(22), pp. 4403-4419, 2003.

[26] Domenikiotis, C., Spiliotopoulos, M., Tsiros, E. \& Dalezios, N.R., Early cotton yield assessment by the use of the NOAA/AVHRR derived Vegetation Condition Index (VCI) in Greece. International Journal of Remote Sensing, 25(14), pp. 2807-2819, 2004.

[27] Goel, N.S., Models of vegetation canopy reflectance and their use in estimation of biophysical parameters from reflectance data. Remote Sens. Rev., 4, pp. 1-212, 1988.

[28] Goel, N.S., Inversion of canopy reflectance models for estimation of biophysical parameters from reflectance data. Theory and Applications of Optical Remote Sensing, ed., G. Asrar, pp. 205-251, 1989.

[29] Verhoef, W., Theory of radiative transfer models applied in optical remote sensing of vegetation canopies. Wageningen: Grafish Service Centrum Van Gils, 1998.

[30] Haboudane, D., Miller, J.R., Pattey, E., Zarco-Tejada, P.J. \& Strachan, I.B., Hyperspectral vegetation indices and novel algorithms for predicting green LAI of crop canopies: Modeling and validation in the context of precision agriculture. Remote Sensing of Environment, 90 (2004), pp. 337-352, 2004.

[31] Vaiopoulos, D., Skianis, G. Aim. \& Nikolakopoulos, K., The contribution of probability theory in assessing the efficiency of two frequently used vegetation indices. International Journal of Remote Sensing, 25(20), pp. 4219-4236, 2004.

[32] Skianis, G. Aim., Vaiopoulos, D. \& Nikolakopoulos, K., A study of the behavior of vegetation index SAVI, based on probability theory. Proceedings of the 7th Panhellenic Congress of the Hellenic Geographical Society, Mytilini, Greece, October 2004, Vol. 1, pp. 41-48, 2004.

[33] Kasnuirangan, K., Ground based measurements for interpretation of vegetation indices over developing countries. Adv. Space Res., 17(8), pp. 31-40, 1996.

[34] Boyd, D.S., Phipps, P.C., Foody, G.M. \& Walsh, R.P.D., Exploring the utility of NOAA AVHRR middle infrared reflectance to monitor the impacts of ENSO-induced drought stress on Sabah rainforests. International Journal of Remote Sensing, 23(2), pp. 5141-5147, 2002.

[35] Chuvieco, E., Martin, M.P. \& Palacios, A., Assessment of different spectral indices in the rednear-infrared spectral domain for burned land discrimination. International Journal of Remote Sensing, 23(23), pp. 5103-5110, 2002.

[36] Peterson, D.L., Price, K.P. \& Martinko, E.A., Discriminating between cool season and warm season grassland cover types in northeastern Kansas. International Journal of Remote Sensing, 23(23), pp. 5015-5130, 2002.

[37] Stroppiana, D., Pinnock, S., Pereira, J.M.C. \& Gregoire, J.M., Radiometric analysis of SPOTVEGETATION images for burnt area detection in Northern Australia. Remote Sensing of Environment, 82, pp. 21-37, 2002.

[38] Tucker, C.J., Red and photographic infrared linear combinations for monitoring vegetation. Remote Sensing of Environment, 8(2), pp. 127-150, 1979. 
[39] Pollock, R.B. \& Kanemasu, E.T., Estimating leaf-area index of wheat with Landsat data. Remote Sensing of Environment, 8(4), pp. 307-312, 1979.

[40] Schowengerdt, R.A., Remote Sensing. Models and Methods for Image Processing. Academic Press, 1997.

[41] Gonzalez, R.C. \& Wintz, P., Digital Image Processing. Addison-Wesley, 1987.

[42] Spiegel, M.R., Probability and Statistics. McGraw-Hill, ESPI, 1977.

[43] Ganas, A. \& Nikolaou, E., Project aperture: synergy of environmental law and remote sensing for land use map production and update. Proceedings of the 6th Panhellenic Geographical Congress, Thessaloniki (Greece), October 3-6, 2002,Vol. II, pp. 127-134, 2002.

[44] Retalis, A., The use of remote sensing for the assessment and monitoring of land cover changes and burned areas in Skiathos Island. Proceedings of the 6th Panhellenic Geographical Congress, Thessaloniki (Greece), October 3-6, 2002, Vol. II, pp. 224-231, 2002.

[45] Nikolakopoulos, K.G., Vaiopoulos, D.A. \& Skianis, G., Aim, Use of Vegetation Indexes and PCA method with remote sensing data for the classification of burnt areas according to how many times they have been burnt. Proceedings of SPIE 10th International Symposium in Remote Sensing, Barcelona, September 8-12, 2003, Vol. 5232, pp. 196-207, 2003.

[46] Sabins, F.F., Remote Sensing: Principles and Interpretation. W. H. Freeman and Company, 1987. 